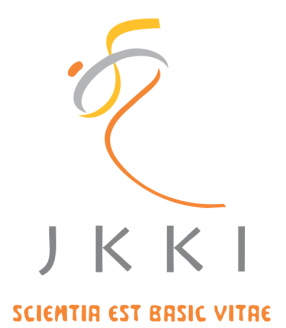

Jurnal Kedokteran dan Kesehatan Indonesia

Indonesian Journal of Medicine and Health

Journal homepage: https://journal.uii.ac.id/JKKI

\title{
Slow deep breathing: Adjuvant physiological intervention to deal with COVID-19
}

Om Lata Bhagat*1

${ }^{1}$ Department of Physiology, All India Institute of Medical Sciences, Jodhpur, India

\begin{tabular}{|c|c|}
\hline & GUEST EDITORIAL \\
\hline ARTICLE INFO & and modern practices which claim to calm \\
\hline $\begin{array}{l}\text { *Corresponding author: } \\
\text { omlatabhagat@gmail.com }\end{array}$ & $\begin{array}{l}\text { Health is considered a positive condition of complete well-being, } \\
\text { reflecting the harmony between all the systems of the body on which }\end{array}$ \\
\hline DOI : 10.20885/JKKI.Vol11.Iss3.art1 & \\
\hline $\begin{array}{l}\text { Copyright @2020 Authors. } \\
\text { This is an open access article } \\
\text { distributed under the terms } \\
\text { of the Creative Commons At- } \\
\text { tribution-NonCommercial } 4.0 \\
\text { International Licence (http:// } \\
\text { creativecommons.org/licences/ } \\
\text { by-nc/4.0/). }\end{array}$ & $\begin{array}{l}\text { feedback mechanism to self-regulate and maintain stability against the } \\
\text { internal and external environment. Any disease or even death can result } \\
\text { from the disturbance in this delicate balance among our body systems. } \\
\text { This concept is also depicted and could be seen in the yogic scriptures; } \\
\text { according to yogic doctrine, diseases or physical ailments (Vyadi) are of } \\
\text { two types Adhija and Anadhija; Adhija are caused during interaction with } \\
\text { the world when the fine balance between man (mind), chitta (psyche), } \\
\text { buddhi (intellect) and sharir (body) are lost. Anadhija includes primary } \\
\text { manifestations on the body due to physical trauma, contagious diseases, } \\
\text { microorganism infestations, etc. Our body has an ability to respond to any } \\
\text { perturbation that disturbs the normalcy in a well-coordinated response } \\
\text { of our physiological systems. Some of the practices are prescribed in our } \\
\text { ancient literature to regain balance and harmony in physiological systems. } \\
\text { Many such techniques have an essential component of slow and deep } \\
\text { breathing. }\end{array}$ \\
\hline
\end{tabular}

The SARS-CoV-2 or coronavirus infection came to us as a significantly stern challenge to our homeostatic response, which has primarily affected the renin-angiotensin-aldosterone system (RAAS) via an interaction with the angiotensin-converting enzyme 2 (ACE2). The studies have shown that the ACE-2 receptor facilitates the entry of corona-virus. The spike proteins on the surface of the SARS-CoV-2 binds to the ACE- 2 receptor on the host cell, leading to the binding of type II transmembrane serine protease (TMPRSS2) and cleaves the ACE-2 receptor. After this, the fusion of viral and cell membranes allows the virus to enter the cell. The activated spike protein, the cleaved ACE-2 receptor, and TMPRSS2 expression increase coronavirus uptake by the host cell. ${ }^{1}$ SARS-CoV-2 has been reported to have a higher affinity for the ACE2 receptor than other coronaviruses. ${ }^{2}$ The ACE2 receptors are present throughout the human body as RAAS regulates electrolyte balance, blood pressure, and fluid volume, thereby involving the brain, lungs, liver, and kidneys. The imbalance thus produced is enhanced by the host response to the virus by inflammation and the proinflammatory cytokines leading to the cytokine storm. ACEinhibitors are common treatment modalities in patients with hypertension, cardiovascular disease, and renal diseases to inhibit RAAS. Also, inhibition of RAAS in obese and diabetics protects against kidney and heart disease. Therefore, people with comorbidities like hypertension, CAD, diabetes, renal disease, metabolic syndrome, estrogen deficiency become easy prey of the COVID-19, and also severity is more. The aging process affects the responses of RAAS, predisposing them to the imbalance and making them 
vulnerable for the COVID-19. ${ }^{3}$

In December 2020, a year passed by since the SARS-CoV-2 was identified as a probable threat with the reports of a cluster of cases of pneumonia of unknown cause, and World Health Organization (WHO) responded quickly with a comprehensive package of guidelines for managing the outbreak of new disease. The world has faced the biggest lockdown during the first half of this year, leading to financial and social disturbances leading to stress and psychological challenges among the unaffected. Now, COVID-19 has reached more than 76 million affected with 1.7 million deaths; still, we don't have a perfect cure or disease prevention strategies. With the new strain of virus being reported in the UK, we don't know when this all will end.

In my opinion, if we delineate the complexities of the homeostatic controls, we may find some answer to this haunting question of finding an effective therapeutic intervention for COVID-19. The effort in understanding and finding the cure and vaccine is reflected in more than 85000 publications only in PMC till date in this year related to COVID-19. The reports have suggested that individuals' physiological immune responses are responsible for a wide range of severity in the people infected with SARS-CoV-2 being asymptomatic to deadly. If we can influence these physiological responses through physiological interventions like physical exercise or breathing practices, we can prevent the unfortunate outcome. People with mild symptoms are advised to manage themselves at home and reach out for medical help only when the severity of illness increases. The serious symptoms include shortness of breath, pressure in the chest, and loss of speech or movement. These guidelines have instilled fear and helplessness in the ordinary person. Undesired emotional or physical situations due to pandemic have resulted in stress. Initially, stress induced was an acute response but becoming chronic as time passing by. This ACTHmediated reaction affects the immune and neuroendocrine systems. It could lead to more burden of diseases like heart disease, rheumatoid arthritis, hypertension, Alzheimer's disease, Parkinson's disease, atherosclerosis, and, finally, unhealthy aging.

As coronavirus is affecting all the spheres of health: physical, mental, and social, we must try to incorporate a simple intervention to enhance our immune system and help ourselves to keep calm and achieve harmony and balance among our physiological systems. Respiration is an 'automatic act' with a higher degree of voluntary control that modulates oscillations in other physiological systems. A study by Nuckowska et. al. (2019) showed that the respiratory component drives the overall increase in blood pressure (BP) and subarachnoid space width oscillations. ${ }^{4}$ A physiological intervention such as breathing exercises like slow and deep breathing at a rate of fewer than seven breaths per minute known for shifting the balance towards the parasympathetic arm of the autonomic nervous system may benefit in these challenging times of the pandemic to reduce the suffering. The slow yogic breathing may serve as a method to draw upon cardio-vagal reserve. These breathing practices can be learned quickly and incorporated into our daily routine; they require only the willingness to perform. Breathing exerts profound influences on cardiovascular autonomic neural outflow as the two systems are coupled reciprocally. The act of slow deep breathing (controlled breathing with a low rate and high tidal volume) modulates cardiac autonomic tone by stimulating baroreceptors, augmenting vagal activity, and reducing sympathetic activity by enhancing central inhibitory rhythms. ${ }^{5}$ Slow deep breathing has a beneficial effect of lowering heart rate and mean arterial pressure that persists after the breathing exercise. Healthy individuals' short-term changes reflect the potential for repetitive breathing exercises evoking long-term changes in cardiovascular control. Even simple volitional breathing shifts cardiac autonomic control towards parasympathetic at the same frequency of spontaneous breathing. Slow and deep breathing also increases alveolar and reduces dead space ventilation, thereby improving ventilation efficiency. Leelarungrayub et al. (2018) has reported the reduction in levels markers of oxidative stress (total antioxidant capacity, glutathione, malondialdehyde, and nitric oxide and inflammatory cytokines such as TNF- $\alpha$ and IL-6) following the 4-week breathing exercise to assume that these may help reduce the severity of COVID-19 (though the research study needs to be undertaken). ${ }^{6}$ Apart from this, slow and deep breathing practices can help minimize and tolerate mild to moderate symptoms. These are reported to produce hypoalgesia, raise oxygen saturation at high altitudes, and prevent gastric dysrhythmias. These respiratory exercises may help people with diabetes and hypertension to maintain better homeostasis as they increase RSA (maximizes around six breaths per minute/ resonant frequency), improves pulmonary gas exchange efficiency, and minimizes cardiac work, and buffers blood 
pressure fluctuations. It primarily affects the autonomic nervous system: Increases vagal activity (vagal tone) and causes parasympathetic dominance, also enhances phasic modulation of sympathetic activity, thus optimises sympathovagal balance. These also improve autonomic responsiveness to physical perturbations (such as standing or physical activities). ${ }^{7}$

A simple, easy to incorporate in the daily routine, physiological intervention of slow deep breathing at the rate of six breath per minute for 10-15 minutes once or twice a day may help the patients of COVID-19 to tolerate mild to moderate symptoms easily. This practice can help unaffected healthy individuals calm their nerves and adjust better to the prevailing situations of this dreaded pandemic.

\section{CONFLICT OF INTERESTS}

The authors have declared that no competing interests.

\section{REFERENCES}

1. Rabi FA, Al Zoubi MS, Al-Nasser AD, Kasasbeh GA, Salameh DM. Sars-cov-2 and coronavirus disease 2019: What we know so far. Pathogens. 2020;9(3).

2. Bohn MK, Hall A, Sepiashvili L, Jung B, Steele S, Adeli K. Pathophysiology of COVID-19: Mechanisms underlying disease severity and progression. Physiology. 2020;35(5):288-301.

3. Sieck GC. Physiology in perspective: A key role of physiology in understanding COVID-19. Physiology. 2020;35(5):286-7.

4. Nuckowska MK, Gruszecki M, Kot J, Wolf J, Guminski W, Frydrychowski AF, et al. Impact of slow breathing on the blood pressure and subarachnoid space width oscillations in humans. Scientific Reports. 2019;9(1).

5. Bhagat OL, Kharya C, Jaryal A, Deepak KK. Acute effects on cardiovascular oscillations during controlled slow yogic breathing. Indian Journal of Medical Research. 2017;145(April):503-12.

6. Leelarungrayub J, Puntumetakul R, Sriboonreung T, Pothasak Y, Klaphajone J. Preliminary study: Comparative effects of lung volume therapy between slow and fast deep-breathing techniques on pulmonary function, respiratory muscle strength, oxidative stress, cytokines, 6-minute walking distance, and quality of life in persons with COPD. International Journal of International Journal of Chronic Obstructive Pulmonary Disease. 2018;13:3909-21.

7. Russo MA, Santarelli DM, O'Rourke D. The physiological effects of slow breathing in the healthy human. Breathe. 2017;13(4):298-309. 